EPJ Web of Conferences 72, 00004 (2014)

DOI: 10.1051/epjconf/20147200004

(C) Owned by the authors, published by EDP Sciences, 2014

\title{
Dark Forces at KLOE/KLOE-2
}

\author{
Francesca Curciarello ${ }^{1,2, a}$ on behalf of KLOE/KLOE-2 Collaborations ${ }^{b}$ \\ ${ }^{1}$ Dipartimento di Fisica e di Scienze della Terra, Università di Messina, F.S. D'Alcontres, 98166 S. Agata- \\ Messina, Italy \\ ${ }^{2}$ INFN-Sezione Catania, I-95123, Catania, Italy
}

\begin{abstract}
Searches for dark matter particles in the GeV mass range and for dark forces are strongly motivated by the numerous striking astrophysical observations recently reported by many experiments.

Flavor factories, like the Frascati $\Phi$-factory DA $\Phi$ NE, are particularly suited to search for the light gauge vector boson, called $\mathrm{U}$ boson, which is thought to mediate an unknown interaction between hypothetical dark matter particles.

By using the KLOE detector, limits on $\mathrm{U}$ boson coupling factor $\varepsilon^{2}$ of the order of $10^{-5} \div$ $10^{-7}$ have been set through the study of the $\phi$ Dalitz decay, the Higgsstrahlung process and $U \gamma$ events. New experiments with the upgraded KLOE detector and the increased luminosity of DA $\Phi N E$ are expected to improve the already set upper limits by a factor of two or better.
\end{abstract}

\footnotetext{
ae-mail: fcurciarello@unime.it

${ }^{b}$ The KLOE/KLOE-2 Collaborations: D. Babusci, I. Balwierz-Pytko, G. Bencivenni, C. Bloise, F. Bossi, P. Branchini, A. Budano, L. Caldeira Balkeståhl, G. Capon, F. Ceradini, P. Ciambrone, F. Curciarello, E. Czerwiński, E. Danè, V. De Leo, E. De Lucia, G. De Robertis, A. De Santis, P. De Simone, A. Di Cicco, A. Di Domenico, C. Di Donato, R. Di Salvo, D. Domenici, O. Erriquez, G. Fanizzi, A. Fantini, G. Felici, S. Fiore, P. Franzini, A. Gajos, P. Gauzzi, G. Giardina, S. Giovannella, E. Graziani, F. Happacher, L. Heijkenskjöld, B. Höistad, M. Jacewicz, T. Johansson, K. Kacprzak, D. Kamińska, A. Kupsc, J. Lee-Franzini, F. Loddo, S. Loffredo, G. Mandaglio, M. Martemianov, M. Martini, M. Mascolo, R. Messi, S. Miscetti, G. Morello, D. Moricciani, P. Moskal, F. Nguyen, A. Palladino, A. Passeri, V. Patera, I. Prado Longhi, A. Ranieri, P. Santangelo, I. Sarra, M. Schioppa, B. Sciascia, M. Silarski, C. Taccini, L. Tortora, G. Venanzoni, W. Wiślicki, M. Wolke, J. Zdebik
}

This is an Open Access article distributed under the terms of the Creative Commons Attribution License 4.0, which permits unrestricted use, distribution, and reproduction in any medium, provided the original work is properly cited. 


\section{Introduction}

The postulation of a non-luminous and nonbaryonic form of matter, called dark matter (DM), dates back to the '30s year of the past century [1-3] when indications of discrepancies in galaxy kinematic have been reported for the first time. Today, the DM existence is well established at galactic [4-7] and cosmological level [8-11]. Nevertheless, evidences for a low energy dark sector weakly coupled to the Standard Model (SM) are equally compelling. In the last years, indeed, many unexpected features in cosmic ray spectra and other astrophysical anomalies have been observed by many experiments. Among the most important we report: the $\mathrm{e}^{+} / \mathrm{e}^{-}$excess in the cosmic ray flux reported by PAMELA [12] and recently confirmed by AMS [14](see Fig. 1); the $511 \mathrm{keV}$ gamma ray signal from galactic center by the INTEGRAL satellite [16]; the total $\mathrm{e}^{+} / \mathrm{e}^{-}$flux measured by Atic [17], Hess [18] and Fermi [19, 20]; the DAMA/LIBRA annual modulation signal[21, 22].

Although each of these discrepancies could find possible astrophysical explanations such as pulsars or supernova shocks [23-26], these observations all together could be explained with the existence of a light (few $\mathrm{GeV}$ ) vector gauge boson belonging to an extra abelian gauge symmetry and mediator of an unknown force between DM particles. This boson should be able to couple with strength $\varepsilon^{2}$ e to ordinary particles by means of kinetic mixing with the SM hypercharge field [27-34]. A tipical value for the kinetic mixing parameter $\varepsilon$ is expected to be of the order $10^{-3}[27,28,33,35-38]$ so that observable effects can be induced at $\mathrm{e}^{+} \mathrm{e}^{-}$colliders $[35,39-$ 41] and fixed target [42-44] or beam dump experiments [45-49].

The $\mathrm{U}$ boson existence could also account for the muon magnetic anomaly discrepancy $a_{\mu}$ giving rise to an additional positive 1-loop contribution that could increase the $a_{\mu}^{S M}$ value, solving the discrepancy for $m_{\mathrm{U}} \sim 10-100 \mathrm{MeV}$ and coupling of $\varepsilon \sim 10^{-3}$ order [50]. Furthermore, a natural hypothesis is that the new symmetry is spontaneously broken by an Higgs-like mecha-
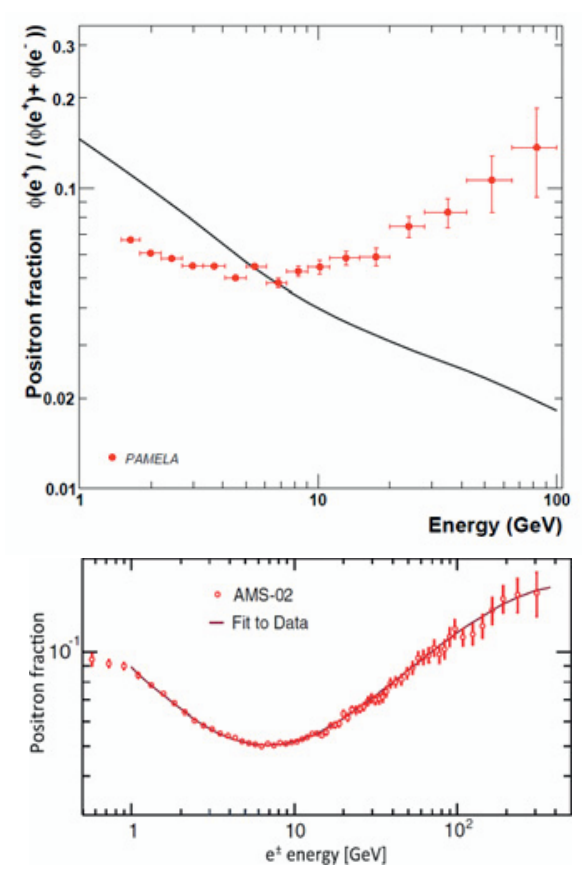

Figure 1. Upper panel: the PAMELA positron fraction compared with theoretical model. The solid line shows a calculation by Moskalenko and Strong [15] for pure secondary production of positrons during the propagation of cosmic-rays in the galaxy. The great discrepancy points to a primary source of positron in the galaxy. Lower panel: the positron fraction measured by AMS and fitted with the minimal model of ref. [14], this model, with diffuse electron and positron components and a common source component, is in very good agreement with data

nism, thus introducing the suggestive hypothesis of the existence of an additional scalar particle.

At KLOE dark forces can be probed by using three different approaches involving meson decays, Higgsstrahlung or radiative processes. In the following we will report on the status of dark forces searches at KLOE/KLOE-2.

\section{DAФNE facility and KLOE Detector}

DAФNE is an $\mathrm{e}^{+} \mathrm{e}^{-}$collider working at the energy $\sqrt{s}=m_{\phi}=1.0195 \mathrm{GeV}$ which is located 
at the National Laboratories of Frascati. The DAФNE Accelerator Complex consists of a linear accelerator, a damping ring, nearly $180 \mathrm{~m}$ of transfer lines, two storage rings that intersect at two points, a beam test area (BTF) and three synchrotron light lines. The KLOE detector is made up of a large cylindrical drift chamber (DC, see Fig. 2), surrounded by a lead scintillating fiber electromagnetic calorimeter (EMC, see Fig. 2). A superconducting coil around the EMC provides a $0.52 \mathrm{~T}$ magnetic field. The EMC provides measurement of photon energies, impact point and an accurate measurement of the time of arrival of particles. The DC is well suited for tracking of the particles and reaction vertex reconstruction. The calorimeter is divided into a barrel and two end-caps and covers $98 \%$ of the solid angle. The modules are read out at both ends by 4880 photo-multipliers. Energy and time resolutions are $\sigma_{E} / E=5.7 \% / \sqrt{E(\mathrm{GeV})}$ and $\sigma_{t}=57 \mathrm{ps} / \sqrt{E(\mathrm{GeV})} \oplus 100 \mathrm{ps}$, respectively. The all-stereo drift chamber, $4 \mathrm{~m}$ in diameter and $3.3 \mathrm{~m}$ long, is made of carbon fiberepoxy composite and operates with a light gas mixture (90\% helium, 10\% isobutane). The position resolutions are $\sigma_{x y} \sim 150 \mu \mathrm{m}$ and $\sigma_{z} \sim$ $2 \mathrm{~mm}$. The momentum resolution is $\sigma_{p_{\perp}} / p_{\perp}$ better than $0.4 \%$ for large angle tracks. Vertices are reconstructed with a spatial resolution of $\sim 3 \mathrm{~mm}$.

\section{$3 \mathrm{U}$ boson searches at KLOE}

KLOE is a well suited place to study the light dark sector for three main reasons: (i) it operates on DAФNE at the $E_{\mathrm{cm}} \approx 1 \mathrm{GeV}$ energy scale; (ii) cross sections for most of the interesting processes involving dark matter at $\mathrm{e}^{+} \mathrm{e}^{-}$ colliders scale with $1 / \mathrm{s}$, this means a factor of 100 with respect to $\mathrm{B}$ factories, almost compensating the loss in integrated luminosity; (iii) it's an ideal place to study some rare meson decays. At KLOE, dark forces are exploited by studying three different processes: light meson decay, $\mathrm{U}$ production through Higgsstrahlung process and $\mathrm{U} \gamma$ events. By investigating these three different processes KLOE has set three upper limits on the kinetic mixing angle $\varepsilon^{2}$.

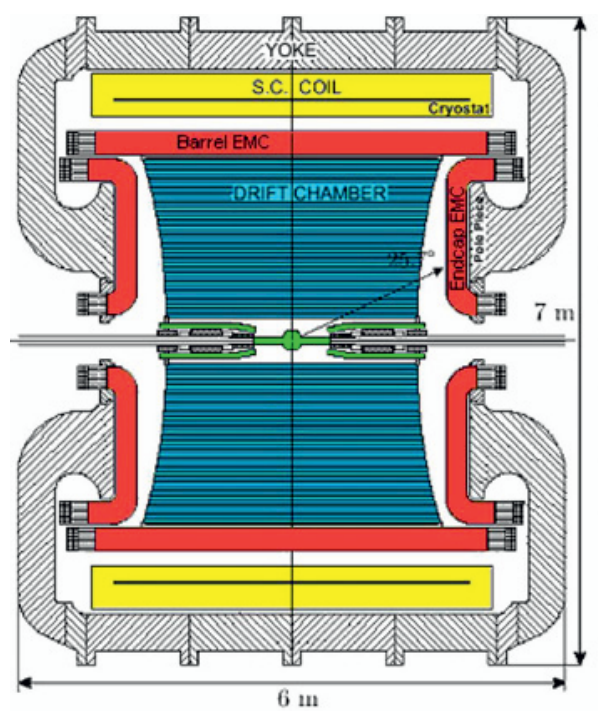

Figure 2. Schematic cross-view of the KLOE apparatus

\subsection{Limit on $\mathrm{U}$ boson production using the $\phi$ Dalitz decay}

The $\mathrm{U}$ can be searched for in vector $(\mathrm{V})$ to pseudoscalar $(\mathrm{P})$ meson decays, with a rate that is $\varepsilon^{2}$ times suppressed with respect to the ordinary $\mathrm{V} \rightarrow \mathrm{P}$ transitions [51]. Since the $\mathrm{U}$ is supposed to decay to $\mathrm{e}^{+} \mathrm{e}^{-}$with a non-negligible branching ratio, $\mathrm{V} \rightarrow \mathrm{PU}$ events will produce a sharp peak in the invariant mass distribution of the electronpositron pair over the continuum background due to Dalitz decay events $\mathrm{V} \rightarrow \mathrm{Pe}^{+} \mathrm{e}^{-}$. Using this approach, KLOE has already published a limit on the existence of the $U$ boson, studying $\phi \rightarrow \eta \mathrm{e}^{+} \mathrm{e}^{-}$decays (see also Fig. 3), where the $\eta$ meson was tagged by its $\pi^{+} \pi^{-} \pi^{0}$ [52]. By applying the analysis procedure described in [52] a first UL on the ratio of $U$ boson coupling constant and fine structure constant of the order of $10^{-5}$ has been derived in the energy range 5$460 \mathrm{MeV}$. This first UL has been then combined, improving sample statistic and background rejection, with the UL derived by tagging the $\eta$ meson by its neutral decay into $3 \pi^{0}$ [53]. The analysis of the decay chain $\phi \rightarrow \eta \mathrm{U}, \eta \rightarrow$ $\pi^{+} \pi^{-} \pi^{0}, \mathrm{U} \rightarrow \mathrm{e}^{+} \mathrm{e}^{-}$, has been performed on a 


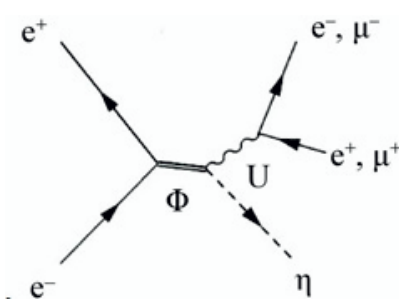

Figure 3. U boson production through Dalitz $\phi$ meson decay

data sample of $1.5 \mathrm{fb}^{-1}$. A first pre-selection is performed by requiring [52]:

- two positive and two negative tracks with point of closest approach to the beam line inside a cylinder around the interaction point (IP), with transverse radius $R_{\mathrm{FV}}=4 \mathrm{~cm}$ and length $Z_{\mathrm{FV}}=20 \mathrm{~cm}$;

- two photon candidates i.e. two energy clusters with $\mathrm{E}>7 \mathrm{MeV}$ not associated to any track, in an angular acceptance $\left|\cos \theta_{\gamma}\right|<0.92$ and in the expected time window for a photon $\left(\mid T_{\gamma}-\right.$ $\left.R_{\gamma} / c \mid<\min \left(5 \sigma_{t}, 2 \mathrm{~ns}\right)\right)$

- best $\pi^{+} \pi^{-} \gamma \gamma$ match to the $\eta$ mass in the pion hypothesis to assign $\pi^{ \pm}$tracks; the other two tracks are then assigned to $\mathrm{e}^{ \pm}$;

- loose cuts of about $\pm 4 \sigma$ 's on $\eta$ and $\pi^{0}$ invariant masses $\left(495<m_{\pi^{+} \pi^{-} \gamma \gamma}<600 \mathrm{MeV}\right.$, $\left.70<M_{\gamma \gamma}<200 \mathrm{MeV}\right)$.

The analysis of the decay channel $\eta \rightarrow 3 \pi^{0}$ is the same as described in [52], with the addition of a cut on the recoil mass to the $\mathrm{e}^{+} \mathrm{e}^{-} \pi^{+} \pi^{-}$system which is expected to be equal to the $\pi^{0}$ mass for signal events. The event selection performed for the $\eta \rightarrow \pi^{0} \pi^{0} \pi^{0}$ decay, on a data sample of $1.7 \mathrm{fb}^{-1}$, requires [53]:

- two opposite charge tracks with point of closest approach to the beam line inside a cylinder, around the interaction point (IP), of $4 \mathrm{~cm}$ transverse radius and $20 \mathrm{~cm}$ length;

- six prompt photon candidates, i.e. energy clusters with $\mathrm{E}>7 \mathrm{MeV}$ not associated to any track, in an angular acceptance $\left|\cos \theta_{\gamma}\right|<$

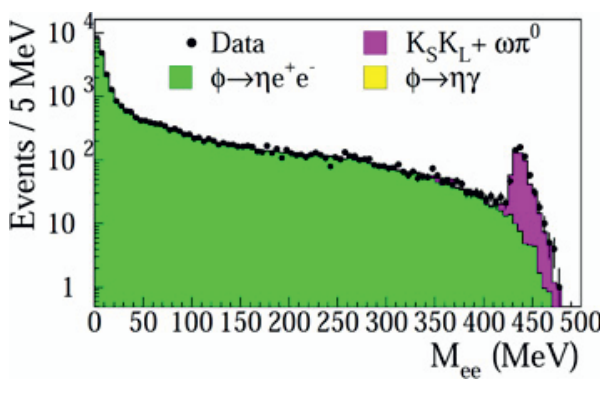

Figure 4. Comparison of experimental and simulated invariant mass $\left(M_{e e}\right)$ distributions for $\phi \rightarrow$ $\eta \mathrm{e}^{+} \mathrm{e}^{-}, \eta \rightarrow 3 \pi^{0}$ events

0.92 and in the expected time window for a photon $\left(\left|T_{\gamma}-R_{\gamma} / c\right|<\min \left(3 \sigma_{\mathrm{t}}, 2 \mathrm{~ns}\right)\right)$;

- a loose cut on the six-photon invariant mass: $400<M_{6 \gamma}<700 \mathrm{MeV}$.

At the end of the analysis chain, 30577 events are selected, with 3\% background contamination (see Fig. 4). The analysis efficiency, defined as the ratio between events surviving analysis cuts and generated events, is $15 \%$ at low $\mathrm{e}^{+} \mathrm{e}^{-}$ invariant masses, increasing up to $30 \%$ at higher $M_{\mathrm{ee}}$ values. The upper limit on the $\mathrm{U}$ boson production in the $\phi \rightarrow \eta \mathrm{U}$ process is obtained through the Confidence level Signal (CLS) technique [54-57] by using the $M_{\mathrm{ee}}$ spectra before background subtraction (see Fig. 5) and by varying the $M_{\mathrm{U}}$ mass, with $1 \mathrm{MeV}$ step, in the range between 5 and $460 \mathrm{MeV}$. Only five bins $(5 \mathrm{MeV}$ width) of the reconstructed $M_{\mathrm{ee}}$ variable, centered at $M_{U}$ are considered. For each channel, the irreducible background, is extracted directly from data after applying a bin-by-bin subtraction of the non-irreducible backgrounds and correcting for the analysis efficiency. The $M_{\mathrm{ee}}$ distribution is then fit (see Fig. 5), excluding the bins used for the upper limit evaluation. $\mathrm{A}^{+} \mathrm{e}^{-}$ invariant mass resolution of about $2 \mathrm{MeV}$ in whole analysis range and a bin-by-bin systematic error on background knowledge have also taken into account in the UL extraction procedure. The limit is extracted both for each $\eta$ decay channel and then combined. For the combined procedure, the CLS evaluation is done by 


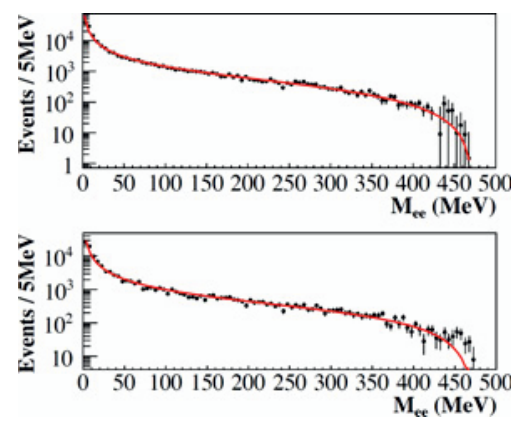

Figure 5. Fit to the corrected $M_{\mathrm{ee}}$ spectrum for the Dalitz decay $\phi \rightarrow \eta \mathrm{e}^{+} \mathrm{e}^{-}$, with $\eta \rightarrow \pi^{0} \pi^{0} \pi^{0}$ (top) and $\eta \rightarrow \pi^{+} \pi^{-} \pi^{0}$ (bottom)

summing values over all bins of the two decay channels, taking into account the different luminosity, efficiency and relative branching ratios of the two samples. In Fig. 6, top, the $\mathrm{UL}$ at $90 \%$ of CL is shown for both eta decay channels. In Fig. 6, bottom, the smoothed combined upper limit on the branching fraction for the process $\phi \rightarrow \eta U, U \rightarrow \mathrm{e}^{+} \mathrm{e}^{-}$, is compared with evaluations from each of the two $\eta$ decay channels. The combined UL on the product $B R(\phi \rightarrow \eta \mathrm{U}) \times B R\left(\mathrm{U} \rightarrow \mathrm{e}^{+} \mathrm{e}^{-}\right)$varies from $10^{-6}$ at small $M_{\mathrm{U}}$ down to $3 \times 10^{-8}$. The exclusion plot in the $\varepsilon^{2}=\alpha^{\prime} / \alpha$ Vs $M_{\mathrm{U}}$ plane has been derived by assuming the following relation [41]:

$$
\begin{aligned}
& \sigma\left(\mathrm{e}^{+} \mathrm{e}^{-} \rightarrow \eta \mathrm{U}\right)=\varepsilon^{2}\left|F_{\phi \eta}\left(m_{\mathrm{U}}^{2}\right)\right|^{2} \times \\
& \frac{\lambda^{\frac{3}{2}}\left(m_{\phi}^{2}, m_{\eta}^{2}, m_{\mathrm{U}}^{2}\right)}{\lambda^{\frac{3}{2}}\left(m_{\phi}^{2}, m_{\eta}^{2}, 0\right)} \sigma\left(\mathrm{e}^{+} \mathrm{e}^{-} \rightarrow \eta \gamma\right),
\end{aligned}
$$

with $\lambda^{\frac{3}{2}}\left(m_{1}^{2}, m_{2}^{2}, m_{3}^{2}\right)=\left[1+m_{3}^{2} /\left(m_{1}^{2}-m_{2}^{2}\right)\right]^{2}-$ $4 m_{1}^{2} m_{3}^{2} /\left(m_{1}^{2}-m_{2}^{2}\right)^{2}$. In Fig. 7 is reported the exclusion plot at $90 \%$ of confidence level derived using both the Vector Meson Dominance (VMD) expectation for the transition form factor slop $\left(b_{\phi \eta} \sim 1 \mathrm{GeV}^{2}\right)$ and the experimental value from SND $\left(b_{\phi \eta} \sim 3.8 \mathrm{GeV}^{2}\right)$. Since the SND values has a $50 \%$ of uncertainty, the UL finally chosen is that more conservative resulting in a limit of: $\alpha^{\prime} / \alpha<1.7 \times 10^{-5}$ for $30<M_{\mathrm{U}}<400 \mathrm{MeV}$, and for the sub-region $50<M_{\mathrm{U}}<210 \mathrm{MeV}$ of $\alpha^{\prime} / \alpha<8.0 \times 10^{-6}$. As

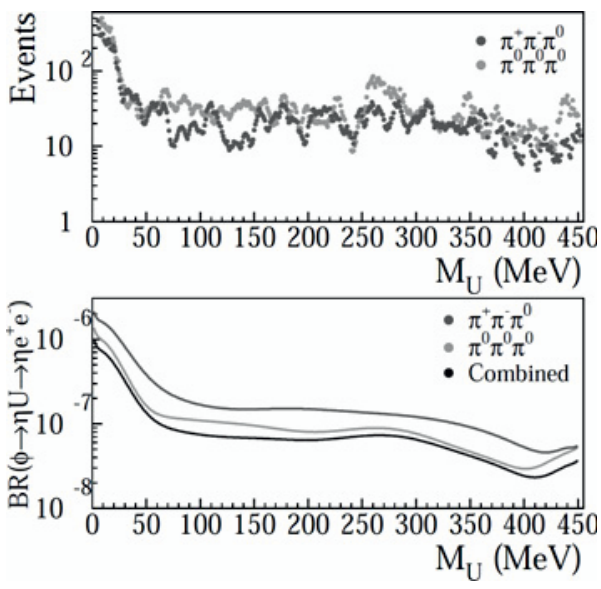

Figure 6. Top: upper limit at $90 \% \mathrm{CL}$ on the number of events for the decay chain $\phi \rightarrow \eta \mathrm{U}, \mathrm{U} \rightarrow$ $\mathrm{e}^{+} \mathrm{e}^{-}$with $\eta \rightarrow \pi^{0} \pi^{0} \pi^{0}$ and $\eta \rightarrow \pi^{+} \pi^{-} \pi^{0}$. Bottom: smoothed upper limit at $90 \% \mathrm{CL}$ on $B R(\phi \rightarrow$ $\eta \mathrm{U}) \times B R\left(\mathrm{U} \rightarrow \mathrm{e}^{+} \mathrm{e}^{-}\right)$, obtained separately for the two $\eta$ decay channels and from the combined analysis

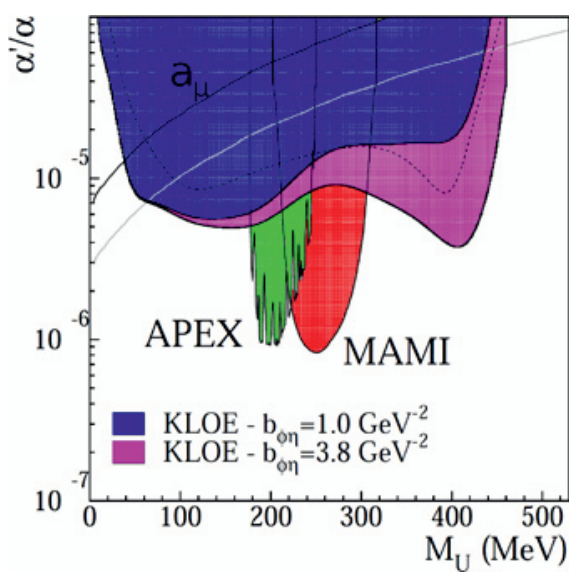

Figure 7. Exclusion plot at $90 \%$ CL for the parameter $\alpha^{\prime} / \alpha=\varepsilon^{2}$, compared with existing limits from the muon anomalous magnetic moment (black line), from MAMI/A1 (red area) and APEX experiments (green area). The gray line represents the expected values of the $U$ boson parameters needed to explain the observed discrepancy between measured and calculated $a_{\mu}$ values. The dotted line is the previous KLOE result, obtained with the $\eta \rightarrow \pi^{+} \pi^{-} \pi^{0}$. For the recent experimental UL in the mass region below $100 \mathrm{MeV}$ see $[58,59]$. 


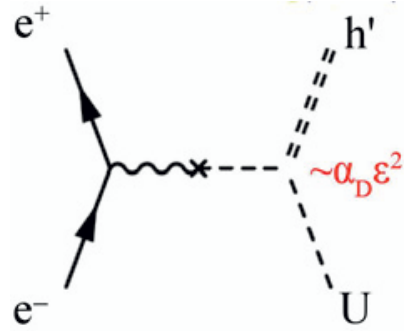

Figure 8. Diagram of the U boson production through higgsstrahalung process

you can see, a wide range of $U$ boson parameters are excluded as possible explanation of the $a_{\mu}$ discrepancy.

\subsection{Search for Higgsstrahlung process}

If the $\mathrm{U}$ boson exists it is also natural to conceive the existence of an elementary Higgs-like boson, the dark higgs $\mathrm{h}^{\prime}$, which spontaneously breaks the extra $U_{D}(1)$ symmetry. Assuming the existence of a higgs' boson, a particularly interesting process from the experimental point of view is the Higgsstrahlung $\mathrm{e}^{+} \mathrm{e}^{-} \rightarrow \mathrm{h}^{\prime} \mathrm{U}$ process (see Fig. 8), which can be observed at KLOE if $m_{\mathrm{U}}+$ $m_{\mathrm{h}^{\prime}}<m_{\phi}$. The signature of this process heavily depends on the $U$ and $h^{\prime}$ mass hierarchy. If the $\mathrm{h}^{\prime}$ is lighter than the $\mathrm{U}$ boson it turns out to be very long-lived (see [40]), so that the signature of the process will be a lepton pair, generated by the $U$ boson decay, plus missing energy. The processes gives many advantages. There are no other physical process with the same signature, the QED $\ell^{+} \ell^{-} \gamma$ background, with the $\gamma$ escaping detection, is strongly suppressed due to high detection efficiency and reaction kinematics, the trigger efficiency is $\sim 99 \%$. More suggestive, such a signature could confirm in one shoot the existence of two new particles. The production cross section of the dark Higgsstrahlung process is proportional to the product $\alpha_{\mathrm{D}} \times \varepsilon^{2}$ and depends on the boson masses [40]. The expected cross section in the KLOE range of interest, for $\varepsilon=10^{-3}$ and $\alpha_{\mathrm{D}}=\alpha_{\mathrm{em}}$, has values up to hundreds of $\mathrm{fb}$. The lifetime of the
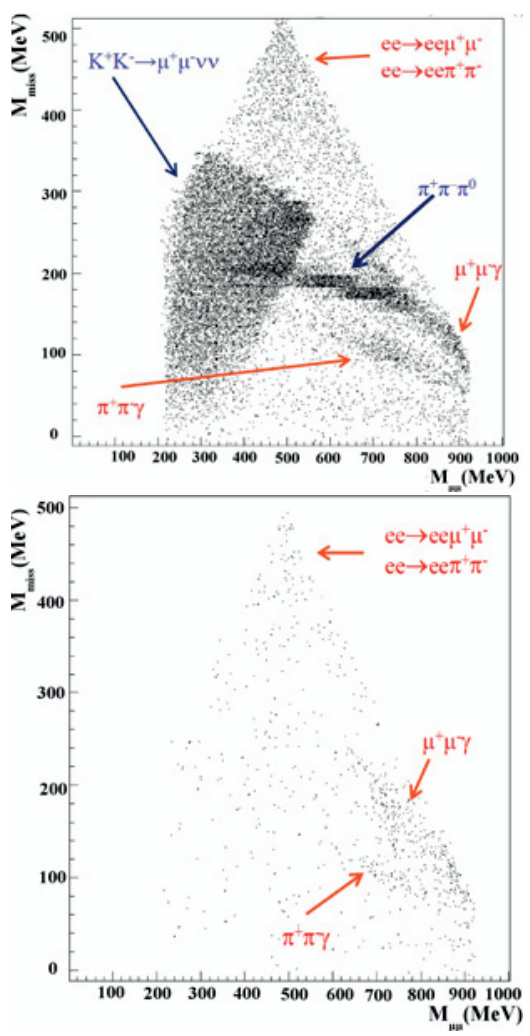

Figure 9. Top panel: $M_{\text {miss }}$ Vs $M_{\mu \mu}$ for on peak sample (1.65 $\mathrm{fb}^{-1}$ integrated luminosity). Bottom panel: $M_{\text {miss }}$ Vs $M_{\mu \mu}$ for the off peak sample, $\left(0.2 \mathrm{fb}^{-1}\right.$ integrated luminosity)

dark Higgs boson depends on the kinetic mixing parameter $\varepsilon$, the boson masses $m_{\mathrm{h}^{\prime}}$ and $m_{\mathrm{U}}$ and the dark coupling constant $\alpha_{\mathrm{D}}$. For masses of the order of $100 \mathrm{MeV}$ and $\alpha_{\mathrm{D}}=\alpha_{\mathrm{em}}$ the dark Higgs boson lifetime would be $\sim 5 \mu$ s for $\varepsilon=10^{-3}$, corresponding, for KLOE energies, to a decay length of $\sim 100 \mathrm{~m}$. The dark Higgs boson would be invisible up to $\varepsilon \sim 10^{-2} \div 10^{-1}$, depending on the $\mathrm{h}^{\prime}$ mass. KLOE is studying the Higgsstrahlung process in the energy range $2 m_{\mu}<M_{\mathrm{U}}<1000 \mathrm{MeV}$, by limiting its search to the $\mathrm{e}^{+} \mathrm{e}^{-} \rightarrow \mathrm{h}^{\prime} \mathrm{U}, \mathrm{U} \rightarrow \mu^{+} \mu^{-}, \mathrm{h}^{\prime}$ invisible [60]. Compared to the B-factory case [61], KLOE benefits of the $1 / \mathrm{s}$ factor and of the resonancelike behaviour expected for the production cross section [40]. The analysis has been performed 
by using a data sample of $1.65 \mathrm{fb}^{-1}$ collected at center of mass energy $E_{\mathrm{cm}}$ corresponding to the $\phi$ peak $(\sim 1019 \mathrm{MeV})$ and a data sample of $0.2 \mathrm{fb}^{-1}$ at $E_{\mathrm{cm}}=1000 \mathrm{MeV}$ (off-peak sample). The event selection requires [60]:

- events with only two opposite charge tracks, with a reconstructed vertex inside a $4 \times 30 \mathrm{~cm}$ cylinder around the interaction point;

- each track must have an associated EMC cluster;

- the visible momentum direction has to be in the barrel: $|\cos \theta|<0.75$;

- the momentum of each reconstructed particle must be below $460 \mathrm{MeV}$;

- the modulus of the missing momentum must exceed $40 \mathrm{MeV}$.

The hermeticity and tightness of the electromagnetic calorimeter has been used as a veto to avoid the presence of photons in the event. The event selection then proceeded by applying particle identification (PID) algorithms almost entirely based on the excellent energy and time resolution of the EMC. After the missing energy and the PID selections, a huge background from $\phi \rightarrow \mathrm{K}^{+} \mathrm{K}^{-}, \mathrm{K}^{ \pm} \rightarrow \mu^{ \pm} v$ events survives in the on peak sample. Since Kaons in KLOE have a decay length of $\sim 90 \mathrm{~cm}$, the reconstructed vertex of the extrapolated muon tracks is thus expected to be displaced from the IP, due to the charged kaon lifetime, and with a bad $\chi^{2}$ of the fitting procedure. A cut based on the vertex-IP distance and on the goodness of the track fitting $\chi^{2}$ allowed to reduce the $\mathrm{K}^{ \pm}$background. Events surviving the described selections have been organized in bidimensional histograms with the muon pair mass $M_{\mu \mu}$ and the event missing mass $M_{\text {miss }}$ on the two axes. In order too keep most of the signal in one bin only, a $5 \mathrm{MeV}$ bin width in the $M_{\mu \mu}$ has been chosen while for $M_{\text {miss }}$ a variable binning of 15 , 30 and $50 \mathrm{MeV}$ widths is used. Results are shown in Fig. 9 for the on peak and off peak sample. in the second case of course the huge backgrounds coming from $\phi$ resonant processes are suppressed. No signal signature has been observed and a bayesian UL on number of signal
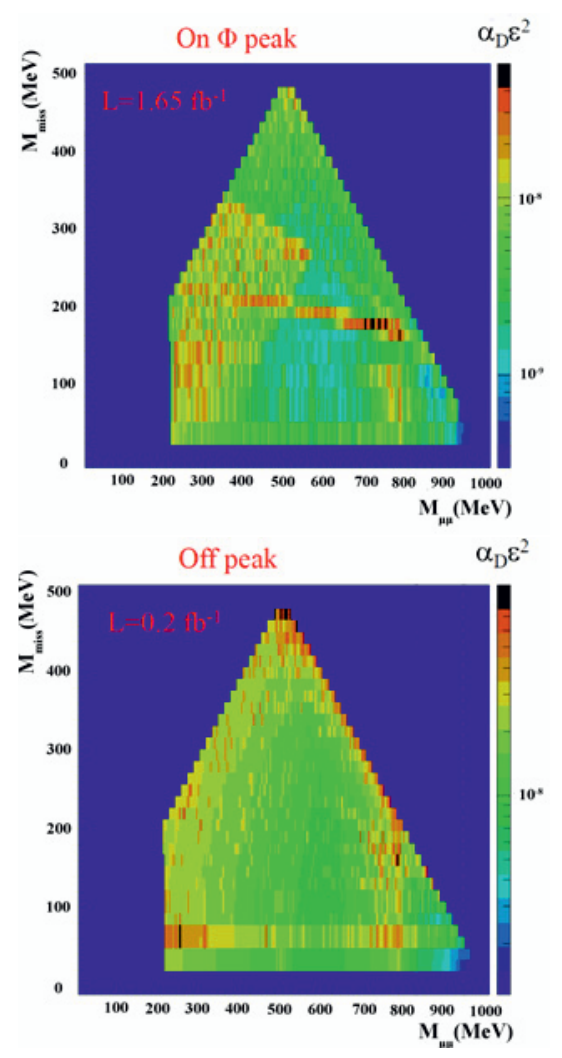

Figure 10. 90\% CL bi-dimensional upper limits in $\alpha_{\mathrm{D}} \times \varepsilon^{2}$ for the on peak sample (upper plot) and off peak sample (bottom plot)

events at $90 \%$ CL has been evaluated, bin-bybin, for the on peak and off peak sample. Results have been translated in terms of $\alpha_{D} \times \varepsilon^{2}$ by using the integrated luminosity information, the signal efficiency and the dark higgsstralung cross section and the branching fraction of the $U \rightarrow \mu^{+} \mu^{-}$decay [40]. The $\alpha_{\mathrm{D}} \times \varepsilon^{2} 90 \% \mathrm{CL}$ limits are shown in Fig. 10, separately for the on peak and off peak sample. These results were then combined by taking into account the different integrated luminosities of the two samples and the expected slightly different signal efficiencies and cross sections due to the different center of mass energies. The combined $90 \%$ CL upper limits projected in the $M_{\mu \mu}$ and $M_{\text {miss }}$ directions and slightly smoothed are shown in 

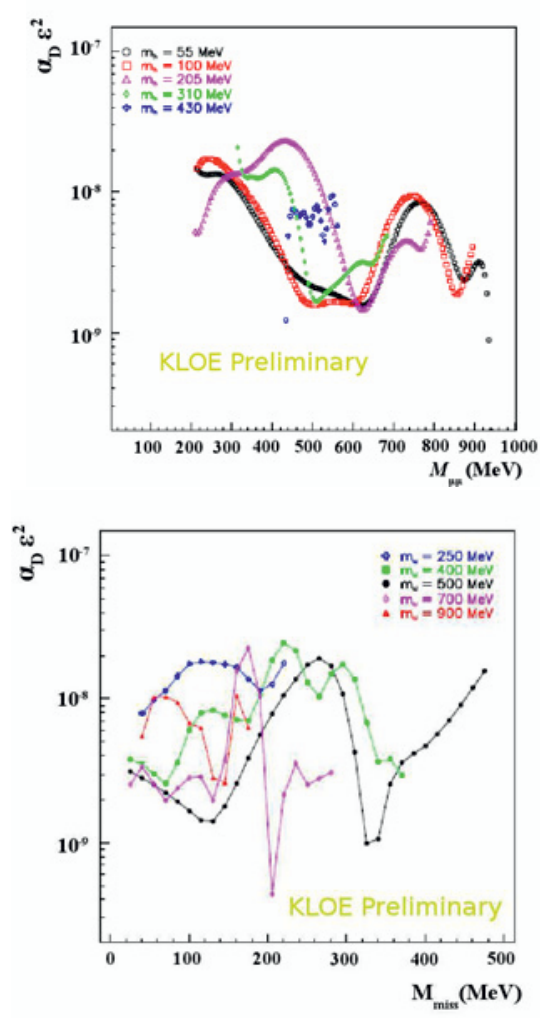

Figure 11. Combined $90 \%$ CL upper limits in $\alpha_{D} \times \varepsilon^{2}$ as a function of $M_{\mu \mu}$ for different values of $m_{\mathrm{h}^{\prime}}$ (upper plot) and as a function of $M_{\text {miss }}$ for different values of $M_{\mathrm{U}}$ (bottom plot plot)

Fig. 11. Values of the order of $10^{-9} \div 10^{-8}$ in $\alpha_{\mathrm{D}} \times \varepsilon^{2}$ are excluded at $90 \% \mathrm{CL}$ for a large range of the dark photon and dark Higgs masses. These limits translate in $\varepsilon \sim 10^{-3}-10^{-4}$ for $\alpha_{\mathrm{D}}=\alpha_{\mathrm{em}}$ and are in agreement and complementary with BaBar results [61] as they refer to the same process in a different final state and phase space region .

\section{3 $\mathrm{U}$ boson search by using the $\mathrm{e}^{+} \mathrm{e}^{-} \rightarrow \mu^{+} \mu^{-} \gamma$ process}

One interesting process to be studied is $\mathrm{e}^{+} \mathrm{e}^{-} \rightarrow$ $\mathrm{U} \gamma, \mathrm{U} \rightarrow \mu^{+} \mu^{-}$shown in Fig. 12. It has the advantage of being a very simple and clean channel, independent of the existence and of the de-

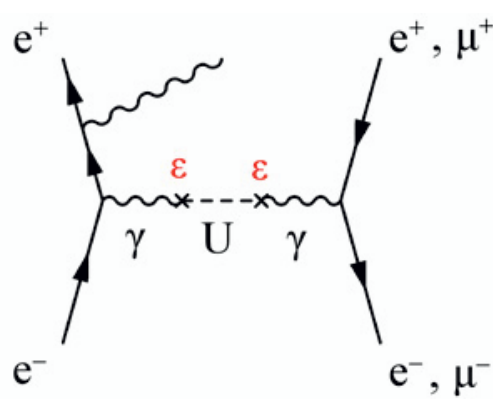

Figure 12. $\mathrm{U}$ boson production at $\mathrm{e}^{+} \mathrm{e}^{-}$colliders through radiative processes

tails of the Higgs sector of the secluded group. A distinctive feature of the expected signal is the appearance of a Breit-Wigner peak in the shape of the invariant mass distribution of the lepton pair induced by the mechanism of photon radiative return and corresponding to $\mathrm{U}$ boson resonant production [35]. Moreover, its expected cross section can be as high as $0.1 \mathrm{pb}$ at DAФNE energies [39].

With the aim to find a $U$ boson peak, we analyzed about $240 \mathrm{pb}^{-1}$ by requiring a small angle event selection $\left(\theta_{\gamma}<15^{\circ}, \theta_{\gamma}>165^{\circ}\right)$ and two charged particles with $50^{\circ}<\theta_{\mu}<$ $130^{\circ}$. This selection gives us a significant reduction of all $\phi$ resonant and final state radiation radiative process background leaving an high initial state radiation statistic signal. The main background channels surviving the selection cuts are represented by radiative Bhabhas $\mathrm{e}^{+} \mathrm{e}^{-} \gamma, \pi^{+} \pi^{-} \gamma$ events and $\pi^{+} \pi^{-} \pi^{0}$. Their contribution has been obtained by fitting data as a superposition of all background contributions plus signal, where $\pi^{+} \pi^{-} \gamma$ and $\pi^{+} \pi^{-} \pi^{0}$ have been estimated with the PHOKHARA Monte Carlo (MC) generator [62] while $\mathrm{e}^{+} \mathrm{e}^{-} \gamma$ have been carefully evaluated from data. Once the measurement/simulation corrections have been applied we extracted the $\mu^{+} \mu^{-} \gamma$ cross section by subtracting the residual background from the observed spectra and dividing it for efficiencies and the integrated luminosity. The $\mu^{+} \mu^{-} \gamma$ absolute cross section, derived by the analysis procedure described above, was then compared with 

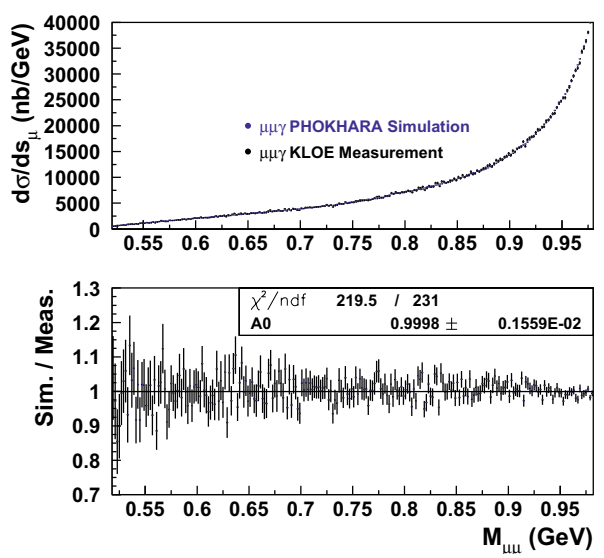

Figure 13. Top: Data-MC comparison of $\mu^{+} \mu^{-} \gamma$ absolute cross section and the QED NLO MC prediction in the 520-980 MeV energy range. Bottom: Ratio of simulated and measured cross sections

the next-to-leading order (NLO) QED simulation from PHOKHARA Event Generator. The comparison is shown in Fig. 13. As it is possible to see an excellent agreement between Data and PHOKHARA MC prediction was achieved. No evidence of $U$ boson peak was found, so an $\mathrm{UL}$ on the kinetic mixing parameter $\varepsilon^{2}$ at $90 \%$ $\mathrm{CL}$ was extracted. The UL on number of signal events has been derived by using the $\mathrm{CL}_{\mathrm{s}}$ technique [54-56] and particularly the TLimit [57] root class. As data input of our procedure we used the observed spectrum and as expected background input the QED NLO MC prediction by PHOKHARA. The signal input has been obtained by generating in step of $2 \mathrm{MeV}$, a gaussian $\mathrm{U}$ boson signal centered at each mass bin, with a sigma value between 1.3-1.8 MeV derived form a $M_{\mu \mu}$ invariant mass resolution study. Also a bin-by-bin systematic error of $1.8-1.3 \%$ on the expected background has been applied to the procedure. The result is reported in Fig. 14 in the whole analysis range at $90 \% \mathrm{CL}$. This result has then been converted in terms of the model parameter $\varepsilon^{2}$ by using the following formula:

$$
\varepsilon^{2}=\frac{\alpha^{\prime}}{\alpha}=\frac{N_{\mathrm{CLS}} /\left(\epsilon_{\mathrm{eff}} \cdot L\right)}{H \cdot I}
$$

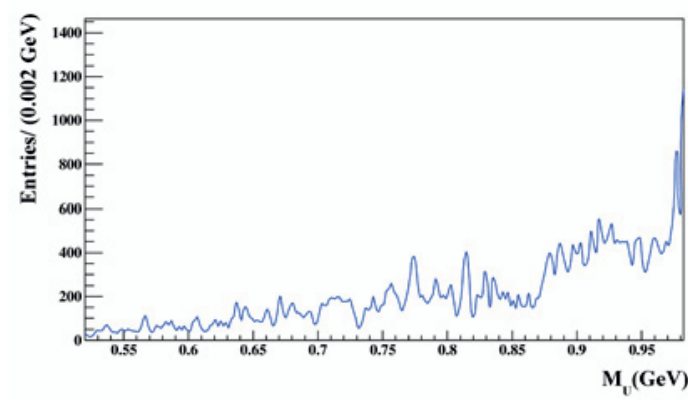

Figure 14. 90\% CL upper limit on number of signal events in the whole investigated range

where $N_{\text {CLS }}$ is the number of entries of signal hypothesis excluded as fluctuations at the $90 \%$ CL reported in Fig. 14. This values have been then corrected for analysis efficiencies and background subtraction; $\epsilon_{\text {eff }}$ are the acceptance corrections; $L$ is the integrated luminosity $L=$ $239.29 \mathrm{pb}^{-1} ; H$ is the radiator function given by:

$$
H=\frac{\mathrm{d} \sigma_{\mu^{+} \mu^{-} \gamma} / \mathrm{d} s_{\mu}}{\sigma\left(\mathrm{e}^{+} \mathrm{e}^{-} \rightarrow \mu^{+} \mu^{-}, s_{\mu}\right)},
$$

where $\mathrm{d} \sigma_{\mu^{+} \mu^{-} \gamma} / \mathrm{d} s_{\mu}$ is the partial cross section of $\mathrm{e}^{+} \mathrm{e}^{-} \rightarrow \mu^{+} \mu^{-} \gamma, s_{\mu}$ is the invariant mass of muons, $\sigma\left(\mathrm{e}^{+} \mathrm{e}^{-} \rightarrow \mu^{+} \mu^{-}, s_{\mu}\right)$ is the total cross section of $\mathrm{e}^{+} \mathrm{e}^{-} \rightarrow \mu^{+} \mu^{-}$process; $I$ is given by the following integral:

$$
I=\int_{i} \sigma_{U}^{\mu \mu} \mathrm{d} s_{i},
$$

where $\sigma_{\mathrm{U}}^{\mu \mu}=\sigma\left(\mathrm{e}^{+} \mathrm{e}^{-} \rightarrow \mathrm{U} \rightarrow \mu^{+} \mu^{-}, s\right)$ is the total cross section of $\mathrm{U}$ boson production decaying in the $\mu^{+} \mu^{-}$channel, $s=M_{\mathrm{U}}^{2}, i$ is the mass bin number. The above integral has been calculated by making explicit its dependence from the kinetic mixing parameter putting $\varepsilon=1$. The result of the application of this formula is given in Fig. 15 where it is shown the exclusion plot on the kinetic mixing parameter $\varepsilon^{2}$ in the range 520-980 MeV in comparison with the existing limits. In blue we can see our result where it is clearly visible the reduction of the sensitivity due to $\rho$ meson at $\sim 0.77 \mathrm{GeV}$, in red the Mami results [63], in dark green the Apex result 


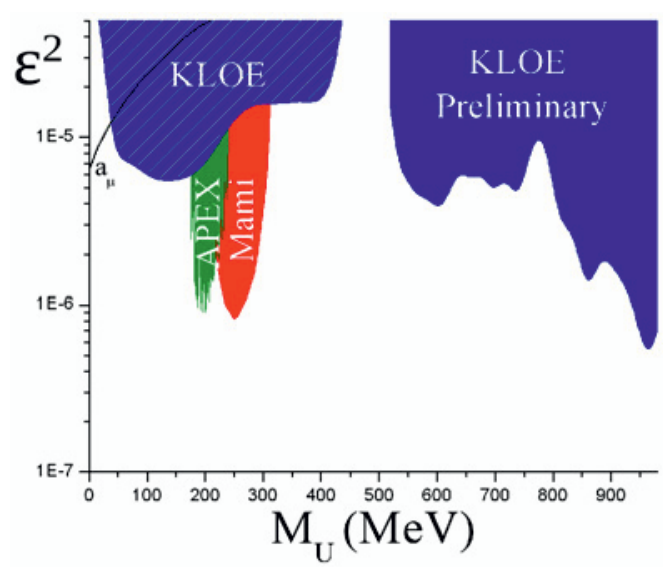

Figure 15. $90 \%$ CL exclusion plot in comparison with existing limits coming from, the Mami (red area) and Apex (green area) experiments and KLOE UL obtained through $\phi$ Dalitz decay. The black line represents $U$ boson parameter values that could explain the $a_{\mu}$ anomaly. WASA limit published in October 2013 is not shown.

[64] and in lined blue the KLOE UL in the region 5-460 MeV calculated using the Dalitz $\phi$ decay $[52,53]$. The black line represents the $\varepsilon^{2}$ values consistent with a $\mathrm{U}$ boson contribution to the muon magnetic moment anomaly $a_{\mu}$.

\section{Conclusions}

KLOE/KLOE2 experiment at DAФNE facility is an ideal place to search for dark forces in a wide range of masses and by exploiting different production mechanism (U $\gamma$ events, $\phi$ Dalitz decay, Higgsstrahlung process). No U boson evidence in the investigated mass ranges is found and upper limits have been set on kinetic mixing parameter in the range $10^{-5} \div 10^{-7}$ depending on the different processes. KLOE-2 and DAФNE upgrades, especially the inner tracker well suited to detect multi-lepton final state events, will give us the possibility to improve the above shown upper limits by a factor of about 2 or better.

\section{References}

[1] J. Oort, Bull. Astron. Inst. Netherlands 6, 249 (1932)

[2] F. Zwicky, Helvetica Physica Acta 6, 110127 (1933)

[3] F. Zwicky, Astrophys. J. 86, 217 (1937)

[4] G. Bertone, D. Hooper and J. Silk, Phys. Rept. 405, 279-390 (2005)

[5] D. Clowe, M. Bradac, A. H. Gonzalez, M. Markevitch, S. W. Randall et al., Astrophys.J. 648, L109-L113 (2006)

[6] U. G. Briel and J. P. Henry, [arXiv:astroph/9711237] (2007)

[7] The SDSS Collaboration J. K. AdelmanMcCarthy et al., [arXiv:astro-ph/0507711] (2005)

[8] http://map.gsfc.nasa.gov/media/121238/ index.html

[9] http://map.gsfc.nasa.gov/

[10] http://map.gsfc.nasa.gov/news/.

[11] The Planck Collaboration P. Ade et al., [arXiv:1303.5076] (2013)

[12] O. Adriani et al., Nature 458, 607-609 (2009)

[13] O. Adriani et al., Phys. Rev. Lett. 102, 051101 (2009)

[14] M. Aguilar et al. Phys. Rev. Lett. 110, 141102 (2013)

[15] I. V. Moskalenko and A. W. Strong, Astrophys. J. 493, 694-707 (1998)

[16] P. Jean et al., Astronomy Astrophysics 407, L55 (2003)

[17] J. Chang et al., Nature 456, 362 (2008)

[18] F. Aharonian et al., Phys. Rev. Lett. 101, 261104 (2008)

[19] A. A. Abdo et al., Phys. Rev. Lett. 102, 181101 (2009)

[20] The Fermi LAT Collaboration M. Ackermann et al., Phys. Rev. Lett. 108, 011103 (2012)

[21] R. Barnabei et al., Eur. Phys. J. C56, 333 (2008)

[22] R. Bernabei et al., Eur. Phys. J. C67, 39-49 (2010) 
[23] F. A. Aharonian, A. M. Atoyan, and H. J. Volk, Astron. Astrophys. 294, L41 (1995)

[24] L. Zhang and K. S. Cheng, Astron. Astrophys. 368, 1063 (2001)

[25] D. Hooper, P. Blasi, and P. D. Serpico, arXiv:0810.1527, (2008)

[26] H. Yuksel, M. D. Kistler, and T. Stanev, Phys. Rev. Lett. 103, 051101 (2009)

[27] B. Holdom, Phys. Lett., B166, 196 (1986)

[28] C. Boehm, P. Fayet, Phys. Lett. B683 , 259 (2004)

[29] M. Pospelov, A. Ritz, M.B. Voloshin, Phys. Lett. B662, 53-61 (2008)

[30] M. Pospelov and A. Ritz, Phys. Lett. B671, 391-397 (2009)

[31] N. Arkani-Hamed, D.P. Finkbeiner, T.R. Slatyer et al., Phys. Rev. D79, 015014 (2009)

[32] I. Cholis, G. Dobler, D.P. Finkbeiner et al., Phys. Rev. D80, 123518 (2009)

[33] M. Pospelov, A. Ritz, Phys. Lett. B671, 391 (2009) 1502

[34] Y. Mambrini, J. Cosmol. Astropart. Phys., 1009, 022 (2010)

[35] L. Barzè et al., Eur. Phys. J. C71, 1680 (2011)

[36] K. R. Dienes, C. F. Kolda and J. MarchRussell, Nucl.Phys. B492, 104-118 (1997)

[37] M. Cicoli, M. Goodsell, J. Jaeckel and A. Ringwald, JHEP 1107, 114 (2011)

[38] C. P. Burgess, J. P. Conlon, L.-Y. Hung, C. H. Kom, A. Maharana and F. Quevedo, JHEP 0807, 073 (2008)

[39] R. Essig, P. Schuster, N. Toro, Phys. Rev. D80, 015003 (2009)

[40] B. Batell et al., Phys.Rev. D79, 11508 (2009)

[41] M. Reece and L.T. Wang, JHEP 0907, 051 (2009)

[42] J. D. Bjorken, R. Essig, P. Schuster and N. Toro, Phys. Rev. D80, 075018 (2009)

[43] B. Batell, M. Pospelov and A. Ritz, Phys. Rev. D80, 095024 (2009)

[44] R. Essig, P. Schuster, N. Toro and B. Wojtsekhowski, JHEP 1102, 009 (2011)
[45] E. Riordan, M. Krasny, K. Lang, P. De Barbaro, A. Bodek et al., Phys.Rev.Lett. 59, 755758 (1987)

[46] J. D. Bjorken, S. Ecklund, W. R. Nelson, A. Abashian, C. Church, B. Lu, L. W. Mo, T. A. Nunamaker and P. Rassmann, Phys.Rev. D38, 3375 (1988)

[47] M. Davier and H. Nguyen Ngoc, Phys. Lett. B229, 150 (1989)

[48] A. Bross, M. Crisler, S. H. Pordes, J. Volk, S. Errede and J. Wrbanek, Phys.Rev.Lett. 67, 2942-2945 (1991)

[49] J. Blumlein and J. Brunner, Phys. Lett. B701, 155-159 (2011)

[50] M. Pospelov, Phys. Rev. D80, 095002 (2009)

[51] M. Reece, L.T. Wang, JHEP 07, 051 (2009)

[52] F. Archilli et al., Phys.Lett B706, 251-255 (2012)

[53] D. Babusci et al., Phys. Lett. B720, 111115 (2013)

[54] G. J. Feldman and R. D. Cousins, Phys. ReV D57, 3873 (1998)

[55] T. Junk, Nucl. Instr. Meth. A434, 435 (1999)

[56] A. L. Read, J. Phys. G: Nucl. Part. Phys. 28, 2693-2704 (2002)

[57] http://root.cern.ch/root/html/TLimit.html

[58] P. Adlarson et al., Phys. Lett. B726, 187193 (2013)

[59] G. Agakishiev et al., arXiv:1311.0216

[60] E. Graziani, Physics Series LVI, (2012) Dark Forces at Accelerators Conference, October 16-19 (2012)

[61] J.P. Lees et al. (BaBar Collab.) Phys. Rev. Lett. 108, 211801 (2012)

[62] H. Czyż, A. Grzelinska, J.H. Kühn, G. Rodrigo, Eur. Phys. J. C 39, 411 (2005)

[63] M. Merkel et al., Phys. Rev. Lett. 106, 251802 (2011)

[64] S. Abrahamyan et al., Phys. Rev. Lett. 107, 191804 (2011) 